Homology, Homotopy and Applications, vol.12(2), 2010, pp.231-244

\title{
PATH CATEGORIES AND RESOLUTIONS
}

\author{
J.F. JARDINE \\ (communicated by Gunnar Carlsson)
}

\begin{abstract}
Path categories are defined, and their basic properties are described, for simplicial and cubical sets. A calculational method for describing the path category $P(K)$ of a finite oriented simplicial complex $K$ is introduced, which involves a finite 2-category which can be specified by generators and relations. This method specializes to higher dimensional automata via the triangulation functor from cubical to simplicial sets, and leads to calculations of their associated execution paths.
\end{abstract}

\section{Introduction}

The path category $P(X)$ of a simplicial set $X$ defines a functor $X \mapsto P(X)$ which is left adjoint to the nerve functor $B$ : cat $\rightarrow s$ Set. The first section of this paper contains an elementary description of this functor, with some of its basic properties. This construction specializes to a path category functor for cubical sets which appears in the second section.

The path category construction is not a standard homotopy invariant for simplicial sets: a map $X \rightarrow Y$ of simplicial sets which induces a homotopy equivalence $|X| \rightarrow|Y|$ of their topological realizations can fail to induce a homotopy equivalence $|B P(X)| \rightarrow$ $|B P(Y)|$. The path category functor is, on the other hand, a strong invariant for Joyal's quasi-category model structure for simplicial sets $[\mathbf{5}],[\mathbf{6}]$ and is, more generally, a theoretical building block for various approaches to higher category theory.

But there is a question: given a simplicial set $X$ with vertices $x$ and $y$, how do you "compute" the morphism set $P(X)(x, y)$ ? Is it empty or not? When is it finite? What does it mean for this set to have more than one element? The purpose of this paper is to display techniques which give answers to these questions in specific cases of interest.

The first answers are counterintuitive from the point of view of ordinary homotopy theory, even for elementary examples. The path category $P(X)$ only sees the 2-skeleton of a space $X$ (as one might expect), and its morphism sets detect 2dimensional holes in $X$. But they also detect missing 2 -simplices in spaces which are contractible in the usual sense: it is shown here (in the first section) that the

This research was supported by NSERC.

Received May 15, 2009, revised June 27, 2010; published on October 8, 2010.

2000 Mathematics Subject Classification: 55U10, 18D05, 68Q85.

Key words and phrases: path category, 2-category, resolutions, higher dimensional automata.

This article is available at http://intlpress.com/HHA/v12/n2/a8

Copyright (c) 2010, International Press. Permission to copy for private use granted. 
path categories of the outer horns $\Lambda_{0}^{3}, \Lambda_{3}^{3}$ are not posets, while the path categories of all inner horns are posets. The non-triviality of the category $P\left(\Lambda_{0}^{3}\right)$ effectively means that the path category detects sources in finite oriented simplicial complexes, and the non-triviality of $P\left(\Lambda_{3}^{3}\right)$ means that the functor detects sinks - see Example 1.6.

Joyal's quasi-category model structure gives methods for identifying $P(X)$ up to equivalence of categories, since every quasi-category weak equivalence $X \rightarrow Y$ induces an equivalence of categories $P(X) \rightarrow P(Y)$. This is useful for dealing with global qualitative questions, but one wants some device for determining the morphism sets $P(X)(x, y)$ explicitly.

The approach taken in this paper, in Sections 3 and 4, is to transfer the question to simplicial categories (or rather, categories enriched in simplicial sets), in which ordinary categories $C$ have resolutions $C_{s}$. Such resolutions have been used for some time in homotopy coherence theory: a simplicial functor defined on $C_{s}$ is used to specify a diagram on $C$ which is functorial, not on the nose in general, but up to some coherent family of homotopies.

Gluing copies of the resolutions $\mathbf{n}_{s}$ of the ordinal number posets $\mathbf{n}$ together along the incidence relations of the simplices $\Delta^{n} \rightarrow X$ defines a simplicial category $P(X)_{\infty}$ and a map $P(X)_{\infty} \rightarrow P(X)$ such that the morphism set $P(X)(x, y)$ is the set of path components of a simplicial set $P_{\infty}(X)(x, y)$ (Lemma 3.1). This means that the question of "computing" $P(X)(x, y)$ can then be approached by studying the ordinary homotopy type of the space $P_{\infty}(X)(x, y)$.

So far, this method works best for finite simplicial complexes $K \subset \Delta^{N}$. The simplicial category morphism $P_{\infty}(K) \rightarrow \mathbf{N}_{s}$ has a 2-category image $P_{2}^{\prime}(K) \subset \mathbf{N}_{s}$ with path component category $P(K)$. Throwing away degenerate paths gives a subobject $N P_{2}^{\prime}(K) \subset P_{2}^{\prime}(K)$ with the same homotopy type.

The 2-category $N P_{2}^{\prime}(K)$ has morphism categories $N^{\prime} P_{2}(K)(v, w)$ which are finite, and have explicit rules for constructing both their object and morphism sets, starting from the simplices of $K$. Furthermore, there is a bijection

$$
\pi_{0} N P_{2}^{\prime}(K)(v, w) \cong P(K)(v, w) .
$$

The isomorphism (1) is a consequence of Theorem 4.4, which is the main result of this paper.

The methods on display here can be used to construct an algorithm to completely determine the categories $N P^{\prime}(K)(v, w)$ and their sets $P(K)(v, w)$ of path components, starting from a list of generating simplices of the finite oriented complex $K$. This algorithm has been successfully implemented in various programming languages as a result of ongoing joint work with Graham Denham and Michael Misamore, and I thank them both for their contributions.

Here is a reason to care: Pratt's higher dimensional automata [7], [2] are finite cubical complexes. These are precisely the subobjects $K \subset \square^{n}$ of the standard $n$ cells $\square^{n}$ which are the building blocks for the category of cubical sets. These objects $K$ are geometric concurrency models: the vertices of $K$ represent the states of an $n$ processor system, each cell of $K$ corresponds to the simultaneous action of subsets the full collection of processors, and the cells of $\square^{n}$ which are not in $K$ represent areas of shared memory (or other resources) that subcollections of the full set of processors cannot simultaneously occupy or use. 
The morphisms $v \rightarrow w$ in the path category $P(K)$ for the cubical complex $K$ represent execution paths from the state $v$ to the state $w$ within the model, and the main problem for geometric concurrency models of this type is to compute these sets of execution paths. This problem is completely solved for small examples, by the results of this paper and the corresponding algorithm and code.

I would like to thank the referee for some remarks which led to a collection of improvements in the exposition of this paper.

\section{The path category functor}

Suppose that $s$ Set is the category of simplicial sets $[\mathbf{1}]$ and that cat is the category of small categories. The path category functor

$$
P: s \text { Set } \rightarrow \text { cat }
$$

is defined to be the left adjoint of the nerve functor

$$
B: \text { cat } \rightarrow s \text { Set. }
$$

The functor $P$ is constructed by specifying its effect on simplices, and then by taking an appropriate colimit on the simplex category $\Delta / X$ for an arbitrary simplicial set $X$. In effect, there is a bijection

$$
\operatorname{hom}\left(\Delta^{n}, B C\right) \cong \operatorname{hom}(\mathbf{n}, C)
$$

for arbitrary small categories $C$ and ordinal numbers (posets)

$$
\mathbf{n}=\{0,1, \ldots, n\},
$$

so that we set $P\left(\Delta^{n}\right)=\mathbf{n}$. Then for a general simplicial set $X$ we set

$$
P(X)=\underset{\Delta^{\vec{n} \rightarrow X}}{\lim _{\longrightarrow}} \mathbf{n}
$$

where the colimit in cat is indexed by members $\Delta^{n} \rightarrow X$ of the simplex category $\Delta / X$.

Recall [1], [4] that the simplex category $\boldsymbol{\Delta} / X$ has as objects all simplices $\Delta^{n} \rightarrow X$, and its morphisms are the commutative diagrams of simplicial set maps

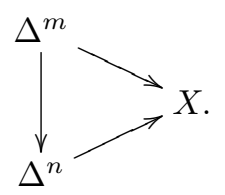

It is well known that the nerve $B C$ of a category $C$ is a 2-coskeleton, which means that the restriction function

$$
\operatorname{hom}(X, B C) \rightarrow \operatorname{hom}\left(\mathrm{sk}_{2}(X), B C\right)
$$

is a bijection for all simplicial sets $X$. This implies the following:

Lemma 1.1. The inclusion $\operatorname{sk}_{2}(X) \subset X$ induces a natural isomorphism

$$
P\left(\operatorname{sk}_{2}(X)\right) \cong P(X)
$$

for all simplicial sets $X$. 
Write $X_{\leqslant 1}$ for the graph $X_{1} \rightrightarrows X_{0}$ given by the truncation of a simplicial set $X$ at level 1. By Lemma 1.1, a simplicial set map $f: X \rightarrow B C$ can be identified with a graph homomorphism $f_{\leqslant 1}: X_{\leqslant 1} \rightarrow C$ which takes each degenerate 1 -simplex $s_{0}(x)$ to the identity $1_{f(x)}$ of the category $C$, and takes all boundaries

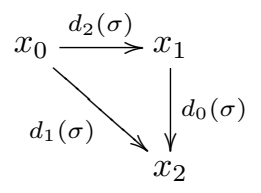

of 2-simplices $\sigma: \Delta^{2} \rightarrow X$ to commutative diagrams

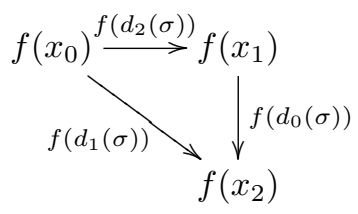

of $C$. The following is an immediate consequence:

Lemma 1.2. The category $P(X)=P\left(\operatorname{sk}_{2}(X)\right)$ is the quotient

$$
F\left(X_{\leqslant 1}\right) / R,
$$

of the free category on the graph $X_{\leqslant 1}$ by the congruence $R$ which is generated by the relations

$$
\begin{aligned}
& s_{0}(x) \sim 1_{x}, x \in X_{0}, \\
& d_{1}(\sigma)=d_{0}(\sigma) d_{2}(\sigma), \sigma \in X_{2} .
\end{aligned}
$$

Recall that a congruence $R$ on a category $C$ is an equivalence relation $\sim$ on each of the morphism sets $C(x, y)$ which is compatible with composition in the sense that if $f \sim f^{\prime}$, then $g f h \sim g f^{\prime} h$ for all morphisms $g, h$.

Lemma 1.2 implies that morphisms $x \rightarrow y$ of the path category $P(X)$ can be represented as paths

$$
x_{0} \stackrel{a_{1}}{\longrightarrow} x_{2} \stackrel{a_{2}}{\longrightarrow} \ldots \stackrel{a_{k}}{\longrightarrow} x_{k}
$$

of 1-simplices in $X$, modulo an equivalence relation defined by degenerate 1-simplices and 2-simplices as in the statement of Lemma 1.2. Composition in $P(X)$ is induced by concatenation of paths.

This path-based description of $P(X)$ is the primary reason for calling it a path category. Some authors $[6]$ say that the category $P(X)$ is the "fundamental category" of $X$.

In all that follows, we will say that a path (2) is non-degenerate if none of the constituent 1-simplices $a_{1}$ is degenerate. Every path in $X$ is equivalent (i.e., congruent) to a non-degenerate path in the description of the path category $P(X)$ given by Lemma 1.2. 
The unit map $\eta: X \rightarrow B P(X)$ for the adjunction is the identity on vertices, and takes a simplex $\sigma: \Delta^{n} \rightarrow X$ to the string of arrows in $P(X)$ determined by the 1simplices

$$
0 \rightarrow 1 \rightarrow \cdots \rightarrow n
$$

in the 1-skeleton of $\Delta^{n}$. We have the following observation:

Lemma 1.3. The natural map $\eta: X \rightarrow B P(X)$ induces a bijection

$$
\pi_{0}(X) \cong \pi_{0}(B P(X))
$$

Here are some other basic properties of the path category construction:

Lemma 1.4. 1) The counit functor $\epsilon: P(B C) \rightarrow C$ is an isomorphism of categories, for all small categories $C$.

2) The projection functors induce a natural isomorphism

$$
P(X \times Y) \stackrel{\cong}{\rightrightarrows} P(X) \times P(Y),
$$

for all simplicial sets $X$ and $Y$.

3) The functor

$$
P\left(\Lambda_{k}^{n}\right) \rightarrow P\left(\Delta^{n}\right) \cong \mathbf{n}
$$

which is induced by the inclusion $\Lambda_{k}^{n} \subset \Delta^{n}$ of the $k^{\text {th }}$ horn $\Lambda_{k}^{n}$ is an isomorphism of categories, provided that $0<k<n$.

Proof. Statements 1) and 3) are proved by showing that the displayed functors between path categories have fully faithful sections. Statement 2) is a consequence of the fact that the product $X \times Y$ is a colimit of products of simplices $\Delta^{n} \times \Delta^{m}$.

Example 1.5. The path category $P\left(\partial \Delta^{2}\right)$ is the universal triangle, while $P\left(\Delta^{2}\right)$ is the universal commutative triangle. The extension problem

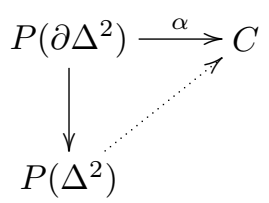

has a solution if and only if the triangle of morphisms in $C$ defined by the functor $\alpha$ commutes. The non-identity morphisms in $P\left(\partial \Delta^{2}\right)$ are represented by the picture

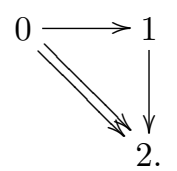

In particular, the two non-trivial morphisms $0 \rightarrow 2$ correspond to the paths

$$
0 \rightarrow 1 \rightarrow 2, \quad 0 \rightarrow 2
$$

of 1-simplices in the simplicial complex $\partial \Delta^{2}$. 
Example 1.6. The inclusion $\partial \Delta^{2} \subset \Lambda_{0}^{3}$ which is induced by the map $d^{0}: \Delta^{2} \rightarrow \Delta^{3}$ induces a fully faithful imbedding

$$
i_{*}: P\left(\partial \Delta^{2}\right) \subset P\left(\Lambda_{0}^{3}\right) .
$$

In particular, there are two distinct morphisms $1 \rightarrow 3$ in the category $P\left(\Lambda_{0}^{3}\right)$. There is exactly one morphism $i \rightarrow j$ for any other pair of vertices $i, j$ of $\Lambda_{0}^{3}$ such that $i \leqslant j$ in the orientation.

The fact that there are two distinct morphisms $1 \rightarrow 3$ (rather than one) in $P\left(\Lambda_{0}^{3}\right)$ is a consequence of the fact that no path from 1 to 3 in $\Lambda_{0}^{3}$ can pass through the vertex 0 , and so the source 0 is detected by the path category $P\left(\Lambda_{0}^{3}\right)$.

A vertex $v$ of a simplicial complex is said to be a source if there are no nondegenerate 1-simplices $\sigma$ with $d_{0}(\sigma)=v$. A vertex $w$ of $K$ is said to be a sink if there are no non-degenerate 1-simplices $\tau$ such that $d_{1}(\tau)=w$.

The inclusion $\partial \Delta^{2} \subset \Lambda_{3}^{3}$ which is induced by the map $d^{3}: \Delta^{2} \rightarrow \Delta^{3}$ induces a fully faithful imbedding

$$
P\left(\partial \Delta^{2}\right) \subset P\left(\Lambda_{3}^{3}\right) .
$$

It follows that there are two morphisms $0 \rightarrow 2$ in $P\left(\Lambda_{3}^{3}\right)$ rather than just one, and so the path category construction detects the sink given by the vertex 3 in $\Lambda_{3}^{3}$. There is exactly one morphism $i \rightarrow j$ for any other pair of vertices $i, j$ of $\Lambda_{3}^{3}$ such that $i \leqslant j$.

Remark 1.7. Any simplicial set map $X \rightarrow Y$ which is a weak equivalence for Joyal's quasi-category model structure for simplicial sets $[\mathbf{6}]$ induces an equivalence of categories $P(X) \rightarrow P(Y)$. Statement 3) of Lemma 1.4 is heavily involved in the proof of this claim, but one also needs to know that a weak equivalence of quasi-categories $X \rightarrow Y$ in Joyal's model structure induces an equivalence $P(X) \rightarrow P(Y)$ of associated path categories.

It follows that the inclusion $\Lambda_{0}^{3} \subset \Delta^{3}$ is not a quasi-category weak equivalence. This inclusion is a weak equivalence for the standard model structure for simplicial sets, since the spaces $\Lambda_{0}^{3}$ and $\Delta^{3}$ are both contractible.

\section{Cubical sets}

The simplicial set $\left(\Delta^{1}\right)^{\times n}$ is isomorphic to the nerve $B\left(\mathbf{1}^{\times n}\right)$ of the categorical hypercube $1^{\times n}$. The category $\mathbf{1}^{\times n}$ is isomorphic to the power set $\mathcal{P}(\underline{n})$ of the set $\underline{n}=\{1, \ldots, n\}$ of $n$ elements, so that a 1 -simplex of $\left(\Delta^{1}\right)^{\times n}$ can be identified with an inclusion $A \subset B$ of subsets of $\underline{n}$.

The top dimensional cells (hypercubes of dimension $n-1$ ) of the boundary subcomplex

$$
\partial\left(\Delta^{1}\right)^{\times n} \subset\left(\Delta^{1}\right)^{\times n}
$$

can be identified with intervals of the form $[\emptyset, \underline{n}-\{i\}]$ and $[\{i\}, \underline{n}]$ in the power set poset, for $1 \leqslant i \leqslant n$.

For $A \subset B \subset \underline{n}$ the interval $[A, B]$ is the poset of subsets $C \subset \underline{n}$ such that $A \subset$ $C \subset B$. There is an isomorphism of posets

$$
[A, B] \cong \mathcal{P}(B-A) \text {. }
$$

It follows that a 1 -simplex $A \subset B$ of $\left(\Delta^{1}\right)^{\times n}$ is in $\partial\left(\Delta^{1}\right)^{\times n}$ if and only if either 
$A \neq \emptyset$ or $B \neq \underline{n}$. This means in particular that $\emptyset \subset \underline{n}$ is the only 1-simplex of $\left(\Delta^{1}\right)^{\times n}$ which is not in the boundary $\partial\left(\Delta^{1}\right)^{\times n}$. A non-degenerate path $\emptyset \rightarrow \underline{n}$ in $\partial\left(\Delta^{1}\right)^{\times n}$ can therefore be identified with a flag of proper inclusions

$$
A: \emptyset=A_{0} \subset A_{1} \subset \cdots \subset A_{k}=\underline{n}
$$

with $A_{1} \neq \underline{n}$.

Suppose that

$$
B: \emptyset=B_{0} \subset B_{1} \subset \cdots \subset B_{m}=\underline{n}
$$

is a second non-degenerate path $\emptyset \rightarrow \underline{n}$ in the boundary. Here are some cases:

1) If $A_{1}$ and $B_{1}$ have a common element $x$ then $A$ is equivalent to the flag

$$
\emptyset \subset\{x\} \subset A_{1} \subset \cdots \subset A_{k}=\underline{n}
$$

while $B$ is equivalent to the flag

$$
\emptyset \subset\{x\} \subset B_{1} \subset \cdots \subset B_{m}=\underline{n} .
$$

The subflags $\{x\} \subset A_{1} \subset \cdots \subset \underline{n}$ and $\{x\} \subset B_{1} \subset \cdots \subset \underline{n}$ are equivalent, because both lie in the face poset $[\{x\}, \underline{n}]$, so that $A$ is equivalent to $B$.

2) Suppose that $A_{1} \cap B_{1}=\emptyset$, and choose $x \in A_{1}$ and $y \in B_{1}$. Then the picture of inclusions

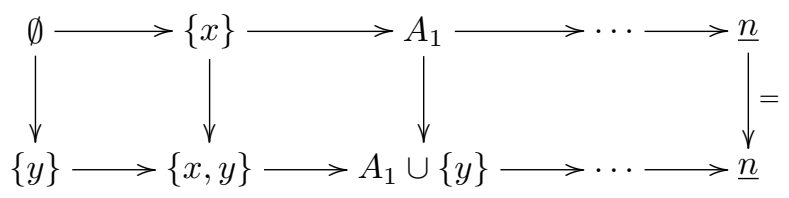

(note that $\{x, y\} \neq \underline{n}$ since $n \geqslant 3$ ) implies that $A \sim A_{x}$ is equivalent to the path

$$
\emptyset \subset\{y\} \subset\{x, y\} \subset A_{1} \cup\{y\} \subset \cdots \subset \underline{n},
$$

which is equivalent to $B_{y} \sim B$. It follows that $A$ is equivalent to $B$.

We have effectively proved:

Lemma 2.1. Suppose that $n \geqslant 3$. Then the inclusion $\partial\left(\Delta^{1}\right)^{\times n} \subset\left(\Delta^{1}\right)^{\times n}$ induces an isomorphism

$$
P\left(\partial\left(\Delta^{1}\right)^{\times n}\right) \cong P\left(\left(\Delta^{1}\right)^{\times n}\right)
$$

of path categories.

Proof. There is a path between any two vertices in the complex $\partial\left(\Delta^{1}\right)^{\times n}$, and

$$
P\left(\left(\Delta^{1}\right)^{\times n}\right) \cong P\left(\Delta^{1}\right)^{\times n} \cong \mathbf{1}^{\times n}
$$

is a poset by Lemma 1.4. It therefore suffices to show that any two paths between a fixed pair of vertices are equivalent.

This will be so for any pair of vertices $A \subset B$ with either $A \neq \emptyset$ or $B \neq \underline{n}$, because pairs of paths of this form are in one of the generating $(n-1)$-cells of $\partial\left(\Delta^{1}\right)^{\times n}$. The cases corresponding to $A=\emptyset$ and $B=\underline{n}$ are dealt with in the paragraphs preceding the statement of the Lemma. 
Recall from [4] that the triangulation $|Y|$ of a cubical set $Y$ is the simplicial set defined by

$$
|Y|=\underset{\square^{n \rightarrow Y}}{\lim _{\longrightarrow}}\left(\Delta^{1}\right)^{\times n},
$$

where the colimit is indexed by objects $\square^{n} \rightarrow Y$ of the cell category $\square / Y$. This functor has a right adjoint $S_{\square}: s$ Set $\rightarrow c$ Set, which is sometimes called the cubical singular functor. The triangulation functor takes the cubical set inclusion $\partial \square^{n} \subset \square^{n}$ to the simplicial set inclusion $\partial\left(\Delta^{1}\right)^{\times n} \subset\left(\Delta^{1}\right)^{\times n}$, up to isomorphism. More generally triangulation takes any finite cubical subcomplex $K \subset \square^{N}$ to a finite simplicial complex $|K|$, where

$$
|K| \subset\left(\Delta^{1}\right)^{\times n} \subset \Delta^{2^{n}-1}
$$

Corollary 2.2. 1) Suppose that the diagram

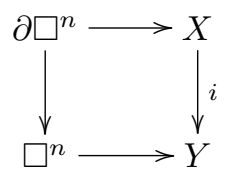

is a pushout in the category of cubical sets, where $n \geqslant 3$. Then the induced map of triangulations $i_{*}:|X| \rightarrow|Y|$ induces an isomorphism of path categories $i_{*}: P(|X|) \cong P(|Y|)$.

2) The inclusion $\operatorname{sk}_{2}(X) \subset X$ of the 2 -skeleton $\operatorname{sk}_{2}(X)$ of a cubical set $X$ induces an isomorphism of path categories $P\left(\left|\mathrm{sk}_{2}(X)\right|\right) \cong P(|X|)$.

To put it a different way, if $Y$ is a cubical set and $X$ is obtained from $Y$ by removing an $n$-cell, where $n \geqslant 3$, then $P(|X|) \cong P(|Y|)$.

I write $P(Y)=P(|Y|)$ for cubical sets $Y$, and say that $P(Y)$ is the path category of the cubical set $Y$. The functor $Y \mapsto P(Y)$ is left adjoint to the cubical nerve functor $C \mapsto B_{\square}(C)$. The cubical nerve $B_{\square}(C)$ of a small category $C$ is the cubical set whose $n$-cells are the functors $\mathbf{1}^{\times n} \rightarrow C$.

The path category $P\left(\partial \square^{2}\right)$ is the universal square diagram while the path category $\left(P \square^{2}\right)$ is the universal commutative square, and the extension problem

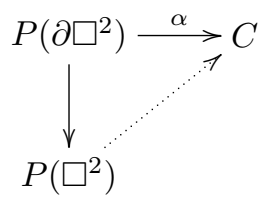

has a solution if and only if the square diagram defined by $\alpha$ commutes. We then have the following analog of Lemma 1.2 for cubical sets:

Lemma 2.3. The path category $P(X)$ for a cubical set $X$ is isomorphic to the free category on the graph $\mathrm{sk}_{1} X$, modulo the relations $b a=a^{\prime} b^{\prime}$ arising from all 2-cells 
$\sigma: \square^{2} \rightarrow X$ with boundary

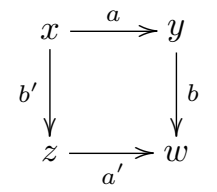

in $X$, as well as $s_{0} x=1_{x}$ for all vertices $x$ of $X$.

\section{Simplicial categories}

In all that follows, a simplicial category will be a category which is enriched in simplicial sets.

We begin by recalling a construction from [1, IX.3.2], [3]. Suppose that $I$ is a small category, and let $a, b$ be objects of $I$. There is a category $I_{s}(a, b)$ whose objects are the strings of arrows $\theta: \mathbf{n} \rightarrow I$ which start at $a$ and end at $b$. A morphism of $I_{s}(a, b)$ is a commutative diagram of functors

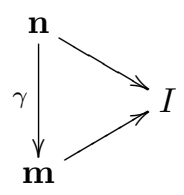

where the functor $\gamma$ is end-point preserving in the sense that $\gamma(0)=0$ and $\gamma(n)=m$. Concatenation of strings defines functors

$$
I_{s}(a, b) \times I_{s}(b, c) \rightarrow I_{s}(a, c)
$$

and $I_{s}$ is therefore a 2-category with 0 -cells the objects of $I$, 1-cells the strings of composable arrows, and 2-cells defined by the end-point preserving functors $\gamma$ above. Taking all classifying spaces $B I_{s}(a, b)$ together gives a simplicial category $B I_{s}$ and a natural map $\epsilon: B I_{s} \rightarrow I$ of simplicial categories (where $I$ is discrete in the simplicial direction).

The map $\epsilon: B I_{s}(a, b) \rightarrow I(a, b)$ is a weak equivalence, since the path component corresponding to the morphism $f: a \rightarrow b$ of $I_{s}(a, b)$ has an initial object given by the 1-simplex $f: \mathbf{1} \rightarrow I$. It follows that the map $\epsilon: B I_{s} \rightarrow I$ is a resolution of the category $I$ in simplicial categories.

Morphisms $\gamma$ as in the diagram (3) are generated by cofaces $d^{j}: \mathbf{k}-\mathbf{1} \rightarrow \mathbf{k}$ such that $0<j<k$, and all codegeneracies $s^{j}: \mathbf{k} \rightarrow \mathbf{k}-\mathbf{1}$, subject to the cosimplicial identities. Each $d^{j}$ is a join (or concatenation) of the form $1 * d^{1} * 1$ for $d^{1}: \mathbf{1} \rightarrow \mathbf{2}$. Similarly, each codegeneracy $s^{j}$ has the form $s^{j}=1 * s^{0} * 1$ for $s^{0}: \mathbf{1} \rightarrow \mathbf{0}$. Among the cosimplicial identities, the only ones which are not satisfied trivially by being in different "join factors" are the cocycle conditions, namely the identities (diagrams)

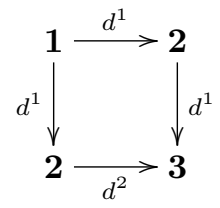


and

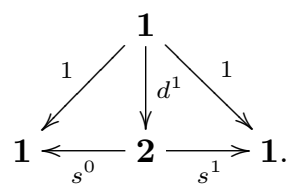

It follows that a 2-functor $f: I_{s} \rightarrow D$ is a lax diagram on $I$ taking values in the 2-category $D$, and that the 2-category $I_{s}$ is the universal lax diagram on the category $I$.

Any simplicial category $E$ has a path component category $\pi_{0}(E)$ whose objects are those of $E$, and such that

$$
\left(\pi_{0}(E)\right)(x, y)=\pi_{0}(E(x, y)) .
$$

Observe that the functors

$$
E_{1} \underset{d_{1}}{\stackrel{d_{0}}{\longrightarrow}} E_{0} \longrightarrow \pi_{0}(E)
$$

form a coequalizer in cat, because all displayed categories have the same sets of objects, and all induced functions on objects are identities.

If $D$ is a 2-category, then forming the classifying spaces $B D(x, y)$ of the morphism categories $D(x, y)$ determines a simplicial category $B D$. If $E$ is a simplicial category, then the path category functor determines categories $P E(x, y)$, and then Lemma 1.4 can be used to show that the categories $P E(x, y)$ define a 2-category $P E$. The functor $E \mapsto P E$ is left adjoint to the functor $D \mapsto B D$.

Now suppose that $X$ is a simplicial set, and write $P_{\infty}(X)$ for the simplicial category which is defined by

$$
P_{\infty}(X)=\underset{\Delta^{n \rightarrow X}}{\lim _{\vec{n}}} B \mathbf{n}_{s},
$$

where the colimit is in the category of simplicial categories. This colimit is formed by taking the colimit in categories in each simplicial degree. I write

$$
P_{2}(X)=P\left(P_{\infty}(X)\right)
$$

to define a functor $P_{2}: s$ Set $\rightarrow 2$ - cat which is left adjoint to the 2-category nerve functor $B_{2}: 2$ - cat $\rightarrow s$ Set. For a 2-category $D$, the $n$-simplices of $B_{2}(D)$ are the 2 category morphisms $\mathbf{n}_{s} \rightarrow D$, or equivalently the lax functors on $\mathbf{n}$ which take values in $D$.

Lemma 3.1. There are isomorphisms of categories

$$
\pi_{0}\left(P_{2}(X)\right) \cong \pi_{0}\left(P_{\infty}(X)\right) \cong P(X)
$$

which are natural in simplicial sets $X$.

Proof. The resolution maps $\epsilon: B\left(\mathbf{n}_{s}\right) \rightarrow \mathbf{n}$ induce isomorphisms $\pi_{0}\left(B\left(\mathbf{n}_{s}\right)\right) \cong \mathbf{n}$, as well as a comparison map

$$
P_{\infty}(X) \rightarrow P(X) .
$$

The functor $D \mapsto \pi_{0}(D)$ commutes with colimits in simplicial categories, and so there 
is an induced isomorphism

$$
\pi_{0}\left(P_{\infty}(X)\right) \cong P(X)
$$

The canonical map

$$
\eta: P_{\infty}(X) \rightarrow B P\left(P_{\infty}(X)\right)=B P_{2}(X)
$$

induces a natural isomorphism of categories

$$
\pi_{0}\left(P_{\infty}(X)\right) \cong \pi_{0}\left(P_{2}(X)\right)
$$

by Lemma 1.3.

\section{Finite simplicial complexes}

Suppose that $K \subset \Delta^{N}$ is a finite simplicial complex. There is a 2-category $P_{2}^{\prime}(K) \subset$ $\mathbf{N}_{s}$ whose path component category is isomorphic to the path category $P(K)$.

Explicitly, the 2-category $P_{2}^{\prime}(K)$ is the subobject of $\mathbf{N}_{s}$ whose objects are the vertices of $K$, and whose 1-cells are the functors $\theta: \mathbf{m} \rightarrow \mathbf{N}$ such that each 1-simplex $\theta(i) \leqslant \theta(i+1)$ is a 1 -simplex of $K$. The category $P_{2}^{\prime}(K)(v, w)$ is the subcategory of $\mathbf{N}_{s}(v, w)$ whose objects are the 1-cells, and which has morphisms generated by all codegeneracies, and all end-point preserving cofaces

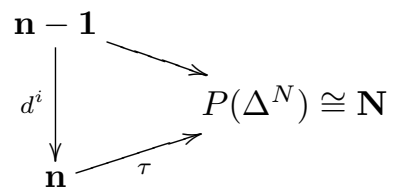

such that the simplex $\tau(i-1) \leqslant \tau(i) \leqslant \tau(i+1)$ is a 2-simplex of $K$.

Alternatively, the 2-category $P_{2}^{\prime}(K)$ as the image in $\mathbf{N}_{s}$ of the 2-category morphism

$$
P_{2}(K) \rightarrow P_{2}\left(\Delta^{N}\right)=\mathbf{N}_{s}
$$

Lemma 4.1. There is a 2-functor

$$
\gamma: P_{2}^{\prime}(K) \rightarrow P(K)
$$

which induces an isomorphism

$$
\pi_{0}\left(P_{2}^{\prime}(K)\right) \cong P(K)
$$

of the path component category $\pi_{0}\left(P_{2}^{\prime}(K)\right)$ of $P_{2}^{\prime}(K)$ with the path category $P(K)$.

Proof. The functor $\gamma$ is defined by the functor $P_{2}^{\prime}(K)_{0} \rightarrow P(K)$ which is the identity on vertices and takes a morphism $\theta: \mathbf{m} \rightarrow \mathbf{N}$ in $P_{2}^{\prime}(K)_{0}($ a path in $K)$ to the morphism $[\theta]$ in $P(K)$ which is represented by $\theta$. Then $\gamma d_{0}=\gamma d_{1}$ for the coequalizer

$$
P_{2}^{\prime}(K)_{1} \underset{d_{1}}{\stackrel{d_{0}}{\longrightarrow}} P_{2}^{\prime}(K)_{0} \longrightarrow \pi_{0}\left(P_{2}^{\prime}(K)\right)
$$

and so there is a canonical induced functor $\gamma_{*}: \pi_{0}\left(P_{2}^{\prime}(K)\right) \rightarrow P(K)$. The category $P(K)$ has the form $P_{2}^{\prime}(K)_{0} / R$ where $R$ is the congruence generated by the 2-simplex and degeneracy relations in $P_{2}^{\prime}(K)_{0}$. The congruence consisting of pairs of morphisms 
with the same image in $\pi_{0}\left(P_{2}^{\prime}(K)\right)$ contains the generating pairs of $R$, and so there is a unique functor $\zeta: P(K) \rightarrow \pi_{0}\left(P_{2}^{\prime}(K)\right)$ such that the diagram

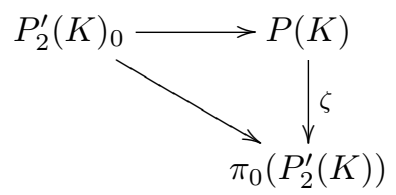

commutes. Then the maps $\gamma_{*} \zeta$ and $\zeta \gamma_{*}$ are identities since the morphisms $P_{2}^{\prime}(K)_{0} \rightarrow$ $P(K)$ and $P_{2}^{\prime}(K)_{0} \rightarrow \pi_{0}\left(P_{2}^{\prime}(K)\right)$ are surjective in objects and morphisms.

Suppose now that $v \leqslant w$ are vertices of $K$, and suppose that $P: \mathbf{n} \rightarrow \mathbf{N}$ is an object of $P_{2}^{\prime}(K)(v, w)$. Then the epi-monic factorization

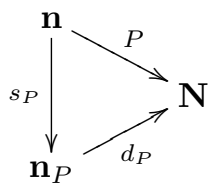

is a canonically defined morphism of $P_{2}^{\prime}(K)(v, w)$ such that $d_{P}$ is a non-degenerate path in $K$ and $s_{P}$ is a co-degeneracy.

Here, a non-degenerate path in $K$ is a path $\mathbf{r} \rightarrow \mathbf{N}$ in $K$ which is non-degenerate as a simplex of $\Delta^{N}$, meaning that it is a monic ordinal number morphism.

Suppose that $i$ is a vertex of $\mathbf{n}$ such that $0<i<n$, and let $\sigma_{i}: \mathbf{2} \rightarrow \mathbf{n}$ be the 2-simplex such that $\sigma_{i}(0)=i-1, \sigma_{i}(1)=i$ and $\sigma_{i}(2)=i+1$. In this notation, the morphisms of $P(K)(v, w)$ are generated in $\mathbf{N}_{s}(v, w)$ by all codegeneracies and by the morphisms

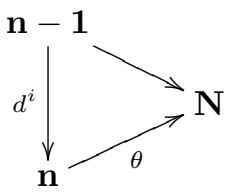

for which the composite

$$
\mathbf{2} \stackrel{\sigma_{i}}{\rightarrow} \mathbf{n} \stackrel{\theta}{\rightarrow} \mathbf{N}
$$

is a 2 -simplex of $K$.

Lemma 4.2. Suppose that $s: \mathbf{n} \rightarrow \mathbf{m}$ is an ordinal number epimorphism and that $0<i<n$. Then either the composite $s \cdot d^{i}$ is an ordinal number epimorphism, or there is a (uniquely determined) commutative diagram

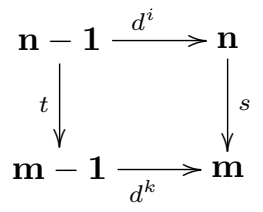

such that $s \cdot \sigma_{i}=\sigma_{k}$. 
Proof. It suffices to assume that $s=s^{j}: \mathbf{n} \rightarrow \mathbf{n}-\mathbf{1}$. Recall the cosimplicial identities

$$
s^{j} d^{i}= \begin{cases}d^{i} s^{j-1} & \text { if } i<j, \\ 1 & \text { if } i=j, j+1, \text { and } \\ d^{i-1} s^{j} & \text { if } i>j+1 .\end{cases}
$$

Then $s^{j}(k)=k$ for $k \leqslant j$ so that $s^{j} \cdot \sigma_{i}=\sigma_{i}$ if $i<j$. Similarly $s^{j}(k)=k-1$ for $k \geqslant j+1$, so that $s^{j} \cdot \sigma_{i}=\sigma_{i-1}$ if $i>j+1$.

Corollary 4.3. Suppose that the morphism

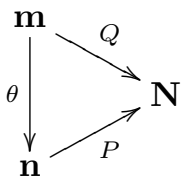

of $P_{2}^{\prime}(K)(x, y)$ is defined by an ordinal number monomorphism $\theta$. Then $\theta$ can be written as a composite of the generators $d^{i}$.

Proof. It suffices to assume that $\theta$ be written as a composite

$$
\theta=s^{j} \cdot d^{i_{k}} \ldots d^{i_{1}}
$$

of generators. In effect, if $\theta=\theta^{\prime} \cdot s$ where $s$ is a codegeneracy, then $s$ is monic as well as epi so that $s=1$. Thus, $\theta$ can be written

$$
\theta=\theta^{\prime \prime} \cdot\left(s^{j} \cdot d^{i_{k}} \ldots d^{i_{1}}\right)
$$

so that the composite

$$
s^{j} \cdot d^{i_{k}} \ldots d^{i_{1}}
$$

is monic.

If $k=1$ and $\theta$ is a monomorphism then $\theta=s^{j} d^{i_{1}}=1$, for otherwise $\theta$ is not monic by the cosimplicial identities.

Suppose that $k>1$. If $s^{j} d^{i_{1}}$ is not the identity, then

$$
s^{j} d^{i_{1}}=d^{i^{\prime}} s^{j^{\prime}}
$$

in $P_{2}^{\prime}(K)(v, w)$ by Lemma 4.2. Then the composite $s^{j^{\prime}} d^{i_{2}} \ldots d^{i_{k}}$ is monic, and can be written as a product of generators $d^{i}$, by the inductive assumption.

Write $N P_{2}^{\prime}(K)(v, w)$ for the full subcategory of $P_{2}^{\prime}(K)(v, w)$ on the non-degenerate paths $\mathbf{n} \rightarrow \mathbf{N}$ in $K$. All morphisms of $N P_{2}^{\prime}(K)(v, w)$ are necessarily ordinal number monomorphisms, and are therefore composites of generating morphisms $d^{i}$ by Corollary 4.3. The non-degenerate paths in $K$ are closed under concatenation, and the inclusions $i: N P_{2}^{\prime}(K)(v, w) \subset P_{2}^{\prime}(K)(v, w)$ together define a 2-functor

$$
i: N P_{2}^{\prime}(K) \subset P_{2}^{\prime}(K) \text {. }
$$

Theorem 4.4. The functors $i: N P_{2}^{\prime}(K)(v, w) \rightarrow P_{2}^{\prime}(K)(v, w)$ are homotopy equivalences, and together induce an isomorphism of path component categories

$$
\pi_{0}\left(N P_{2}^{\prime}(K)\right) \cong \pi_{0}\left(P_{2}^{\prime}(K)\right) \cong P(K) .
$$


Proof. Suppose that

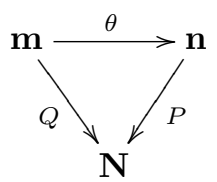

is a morphism of $P_{2}^{\prime}(K)(v, w)$. Then Lemma 4.2 implies that there is a unique morphism $\theta_{*}: d_{Q} \rightarrow d_{P}$ of associated non-degenerate paths in $P_{2}^{\prime}(K)(v, w)$ such that the diagram of ordinal number morphisms

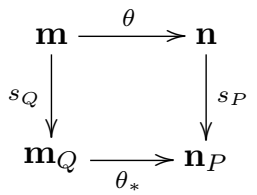

commutes.

Define a functor

$$
r: P_{2}^{\prime}(K)(v, w) \rightarrow N P_{2}^{\prime}(K)(v, w)
$$

by sending $\theta: Q \rightarrow P$ to the morphism $\theta_{*}: d_{Q} \rightarrow d_{P}$. Then $r \cdot i=1$, and the maps $s_{P}$ define a natural transformation $s: 1 \rightarrow i \cdot r$.

\section{References}

[1] P. G. Goerss and J. F. Jardine. Simplicial homotopy theory. Progress in Mathematics, 174. Birkhäuser Verlag, Basel, 1999. xvi+510pp. ISBN:3-7643-6064-X

[2] Eric Goubault. Some geometric perspectives in concurrency theory. Algebraic topological methods in computer science (Stanford, CA, 2001). Homology Homotopy Appl. 5 (2003), no. 2, 95-136.

[3] J. F. Jardine. Modelling homotopy coherence. Category theory 1991 (Montreal, PQ, 1991), 267-283, CMS Conf. Proc., 13, Amer. Math. Soc., Providence, RI, 1992.

[4] J. F. Jardine. Categorical homotopy theory. Homology, Homotopy Appl. 8, (2006), no. 1, 71-144.

[5] A. Joyal. Quasi-categories and Kan complexes. Special volume celebrating the 70th birthday of Professor Max Kelly. J. Pure Appl. Algebra, 175 (2002), no. $1-3,207-222$.

[6] A. Joyal. Notes on quasi-categories, part I. Preprint 2007.

[7] Vaughan R. Pratt. Modeling concurrency with geometry. Proceedings of the 18th ACM SIGPLAN-SIGACT symposium on Principles of programming languages, Orlando, FL (1991), 311-322. ISBN:0-89791-419-8

J.F. Jardine jardine@uwo.ca

Department of Mathematics, University of Western Ontario, London, Ontario, N6A 5B7, Canada 